\title{
Functional Plasticity Triggers Formation and Pruning of Dendritic Spines in Cultured Hippocampal Networks
}

\author{
Miri Goldin, Menahem Segal, and Elena Avignone \\ Department of Neurobiology, The Weizmann Institute, Rehovot 76100, Israel
}

Despite widespread interest in dendritic spines, little is known about the mechanisms responsible for spine formation, retraction, or stabilization. We have now found that a brief exposure of cultured hippocampal neurons to a conditioning medium that favors activation of the NMDA receptor produces long-term modification of their spontaneous network activity. The conditioning protocol enhances correlated activity of neurons in the culture, in a process requiring an increase in $\left[\mathrm{Ca}^{2+}\right]_{i}$ and is associated with both formation of novel dendritic spines and

Novel methods of high-resolution imaging of living dendrites and spines contributed to a radical shift in the century-old view of the spine, from a stable storage site for long-term memory, to a dynamic structure, which can undergo rapid changes in shape and function (Fischer et al., 1998; Segal et al. 2000). The spine is now perceived both as a stable and a plastic structure, and thus it becomes imperative to define the conditions that change spine morphology in association with long-term functional plasticity and dissociate them from ongoing transient variations in spine shape. Dendritic spines in young neurons express considerable motility in different time domains, ranging from small and fast vibrations to slow expansion and retraction (Ziv and Smith, 1996; Fischer et al., 1998; McKinney et al., 1999). These spontaneous movements are reduced in the mature neuron (Dunaevsky et al., 1999), which can still undergo lasting changes in spine shape and density after functional changes. Time lapse photography allows the detection of changes in existing spines or formation of novel ones after exposure of a cultured slice of the hippocampus to a long-term potentiation (LTP) protocol (Engert and Bonhoeffer, 1999), yet there is no evidence that these novel spines are in any way functional, i.e., that they are innervated by afferent fibers and contribute to the enhanced synaptic response. The dissociated culture provides an easy, stable optical access to both the presynaptic and postsynaptic components of the synapse, so that rapid changes in spines can be easily detected (Korkotian and Segal, 1999) in connection with changes in presynaptic elements (Vardinon-Friedman et al., 2000). Unfortunately, except where LTP is recorded between pairs of cells (Tao et al., 2000), there are few established procedures for inducing a reliable and robust LTP in dissociated cultures (Malgaroli and Tsien, 1992; Luscher

\footnotetext{
Received Aug. 17, 2000; revised Oct. 16, 2000; accepted Oct. 19, 2000.

This work was supported by Grant 97/230 from the United States-Israel Binational Science Foundation. We thank Ms. V. Greenberger for the production and maintenance of the cultures and Dr. E. Korkotian for help with the imaging data collection and analysis.

Correspondence should be addressed to Dr. Menahem Segal, Department of Neurobiology, TheWeizmannInstitute,Rehovot76100,Israel.E-mail:menahem.segal@ weizmann.ac.il.

Copyright (C) 2001 Society for Neuroscience $\quad 0270-6474 / 01 / 210186-08 \$ 15.00 / 0$
}

pruning of others. The novel spines are likely to be touched by a presynaptic terminal, labeled with FM4-64 dye, whereas the absence of such terminals increases the likelihood of spine pruning. These results indicate that long-term functional changes are correlated with morphological modifications of dendritic spines of neurons in a network.

Key words: dendritic spines; hippocampus; NMDA; FM4-64; calcium; synapse

et al., 2000). A conventional LTP induction protocol involves an intensive but transient activation of a small set of synapses, which may not favor a large scale formation of dendritic spines. In contrast, continuous activation of a neuronal network, which involves many more synapses, may maximize the likelihood of detection of morphological changes in the cells involved. Taking advantage of the ability to grow neurons in controlled conditions (Segal and Furshpan, 1990), we have developed a protocol in which one can produce a persistent modification of network activity in cultured neurons and monitor simultaneously morphological changes in dendritic spines of these neurons.

\section{MATERIALS AND METHODS}

Culture preparation. Rat pups on day of birth were decapitated, and their brains were removed and placed in a chilled $\left(4^{\circ} \mathrm{C}\right)$, oxygenated Leibovitz L15 medium (Biological Industries, Beit Haemek, Israel) enriched with $0.6 \%$ glucose and Gentamicin $(20 \mu \mathrm{g} / \mathrm{ml}$; Sigma, St. Louis, MO). Bilateral hippocampi of 8-10 pups in each experiment were dissected out and collected in the same medium. Tissue was mechanically dissociated with a fire-polished pasteur pipette and passed to the plating medium consisting of $5 \%$ heat inactivated horse serum (HS), $5 \%$ fetal calf serum (FCS), prepared in MEM-Earl salts (Biological Industries), enriched with $0.6 \%$ glucose, Gentamicin, and $2 \mathrm{~mm}$ glutamax. Approximately $0.3-0.5 \times 10^{6}$ cells in $1 \mathrm{ml}$ of medium were plated in each well of a 24 well plate, onto polylysine-coated round $(13 \mathrm{~mm})$ glass coverslips. A monolayer of glia was grown on the glass for 1-2 weeks before the plating of the neurons (Papa et al., 1995; Murphy and Segal, 1996). Cells were left to grow in the incubator at $37^{\circ} \mathrm{C}, 5 \% \mathrm{CO}_{2}$, for $4 \mathrm{~d}$, then medium was changed to $10 \%$ HS in enriched MEM, plus a mixture of $5^{\prime}$-fluoro-2-deoxyuridineuridine (20 $\mathrm{mg}$ and $50 \mathrm{mg} / \mathrm{ml}$, respectively; Sigma) to block glial proliferation. Four days later the medium was changed once again to $10 \% \mathrm{HS}$ in enriched MEM, and no further changes were made. In some glasses of the plate, $50 \mu \mathrm{M}$ DL-2-amino-7-phosphonovalerate (APV; Tocris, Bristol, UK) was added to the medium every other day, and in a few glasses, $3 \mathrm{~mm}$ $\mathrm{Mg}$ was also added to the medium. There was no clear additional effect of the increased $\mathrm{Mg}$ concentration.

Electrophysiological recording. The cultures were transferred to the recording chamber placed in a Zeiss Axioscope equipped with Nomarski optics, a water immersion lens, and an infrared camera. Single or double whole-cell patch recordings from hippocampal neurons were performed using micropipettes containing (in $\mathrm{mM}$ ): K-gluconate 140, $\mathrm{NaCl} 2$, HEPES 10, EGTA 0.2, Na-GTP 0.3, Mg-ATP 2, and phosphocreatine 10, $\mathrm{pH} 7.4$, having a resistance in the range of 6-12 M 2 . QX314 (2 mM) was added in the pipette solution in voltage-clamp recording. Current and 


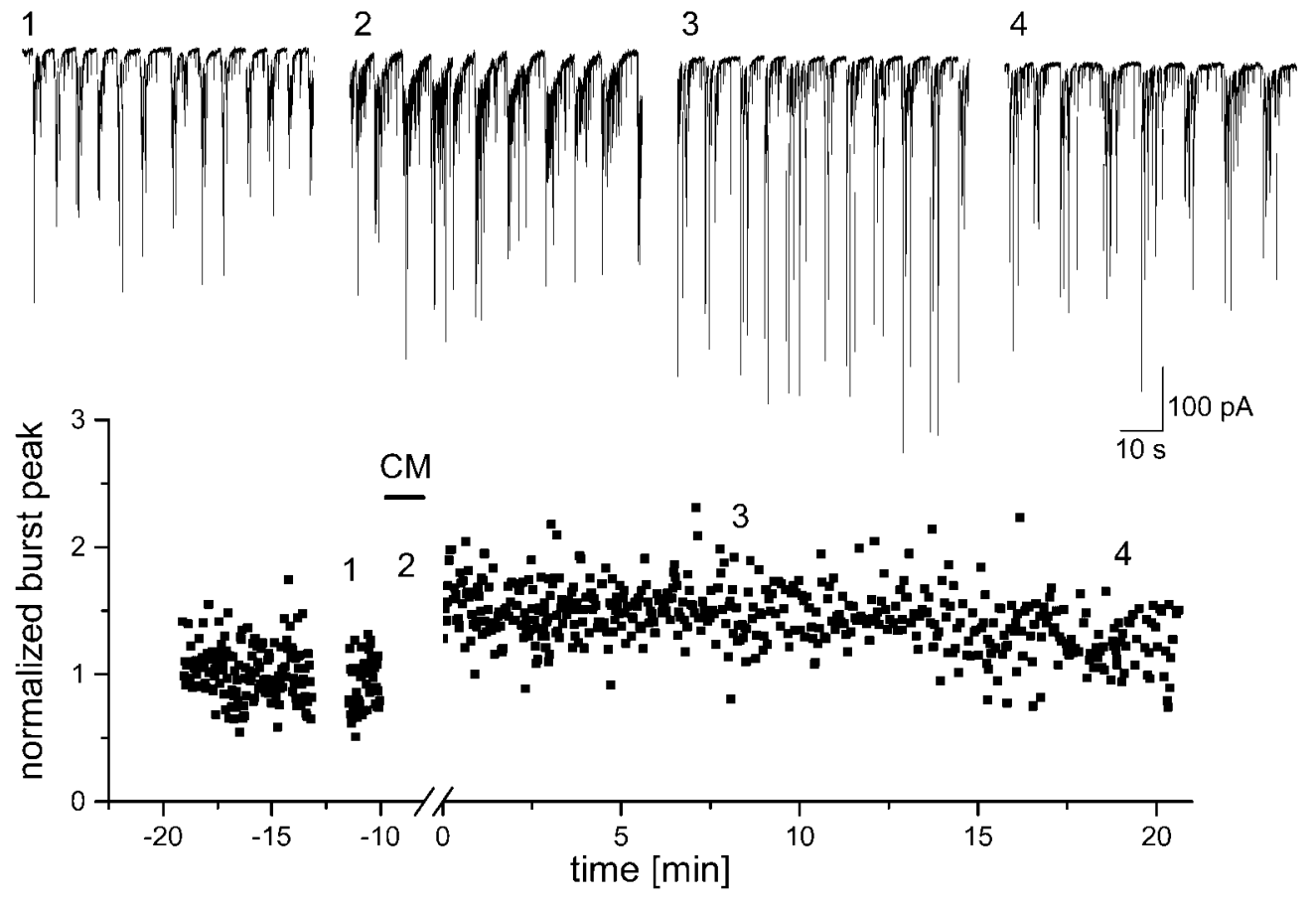

Figure 1. Activity of cells grown in APV-containing medium undergoes longterm modification after brief exposure to a conditioning medium $(C M)$ that favors activation of NMDA receptors. 1-4, Examples of spontaneous activity recorded in APV-treated cultures, before (1), during (2), and at two times after exposure to the CM. Cell was voltage-clamped at -60 $\mathrm{mV}$. Bottom, Continuous recording of burst peaks, normalized to the mean of control periods, during the experiment. A persistent increase in burst size is evident as long as recording could be made from this cell. voltage-clamp recording were performed using Axoclamp 2 and Axopatch 200A (Axon Instruments, Foster City, CA), respectively. Simultaneous recording from two cells in current and voltage clamp were conducted to maximize the chance to detect changes in synaptic and intrinsic currents and also to detect possible changes in firing rates and in the appearance of sustained depolarizations. Voltage-clamped neurons were held at $-60 \mathrm{mV}$, and signals were filtered at $10 \mathrm{kHz}$. Cells in current-clamp were held at approximately $-65 \mathrm{mV}$. Signals were stored in an IBM personal computer using Axon Instruments software. The culture was perfused at a rate of $1 \mathrm{ml} / \mathrm{min}$ at room temperature with standard recording medium containing (in mM): $\mathrm{NaCl} 129, \mathrm{KCl} 4, \mathrm{MgCl}_{2}$ $1, \mathrm{CaCl}_{2} 2$, glucose 10 , and HEPES $10, \mathrm{pH}$ was adjusted to 7.4 with $\mathrm{NaOH}$, and osmolarity to $320 \mathrm{mOsm}$ with sucrose. Perfusion velocity was increased to $4 \mathrm{ml} / \mathrm{min}$ for 3 min during the change of medium to speed up the exchange. During recording from APV-treated cultures, $50 \mu \mathrm{M}$ APV was added to the recording medium. The conditioning medium (CM) was similar to the standard recording medium, except that $\mathrm{Mg}^{2+}$ and APV were omitted, and glycine $(1 \mu \mathrm{M})$ was added to the medium. Activity of cells was compared before and after perfusion with the CM, which was perfused for $2-10 \mathrm{~min}$.

Data were analyzed off-line using pClamp6 (Axon Instruments) and software written in Matlab. The Wilcoxon test was applied for nonparametric statistical analysis to compare data before and after the treatment, and the median of value was considered as representative value of a single cell. The percentage change was calculated as $100 *$ (data after treatment/data before treatment - 1). Data were averaged between different experiments and presented as mean \pm SEM, and $t$ test was applied.

Morphological analysis. Control or APV-treated cultures were washed with the standard recording medium and placed in a recording chamber. Individual cells were injected with calcein $(20 \mathrm{~mm})$ using a sharp micropipette backfilled with K-acetate. The cells were allowed to rest for 30 min, and the chamber was transferred to the stage of a confocal laserscanning microscope (Zeiss 510). The cultures were perfused with the same recording medium as above. Three-dimensional (3D) images of selective dendrites were taken at $30 \mathrm{~min}$ intervals. If no significant movement of spines and filopodia was seen over 30-60 min, the cultures were exposed to the CM for $4 \mathrm{~min}$, followed by wash with the standard recording medium. The same set of dendrites was repeatedly imaged for up to $3 \mathrm{hr}$ after the application of the CM. Image analysis was conducted off-line. Protrusions were categorized into "spines with head", not longer than $3 \mu \mathrm{m}$, "spines with no heads", "stubby spines", $0.5 \mu \mathrm{m}$ or shorter without a spine neck, and "filopodia", thin, longer than $3 \mu \mathrm{m}$ protrusions. The number of spines of all forms was counted in successive dendritic segments of $100-200 \mu \mathrm{m}$ in length. The reliability of the measurements was assessed by a double-blind procedure in which two independent observers analyzed the same subset of images. The two observers yielded highly correlated results. Further analysis of the morphological data were therefore conducted by one trained observer.

When presynaptic terminals were labeled, FM4-64 (2 $\mu \mathrm{M}$; Molecular Probes, Eugene, OR) was applied in a medium containing $90 \mathrm{~mm} \mathrm{KCl}$, replacing equimolar $\mathrm{NaCl}$, for $1 \mathrm{~min}$, followed by an extensive wash in a Ca-free medium, followed by wash in regular medium. FM4-64 labeled puncta were easily detected. These puncta were maintained after extensive wash in $\mathrm{Ca}$-free medium, but disappeared within minutes after wash in normal medium. When FM4-64-labeled particles were imaged, the two-channel option of the confocal microscope was used to take simultaneous images with excitation wavelengths of 488 and $543 \mathrm{~nm}$.

\section{RESULTS}

\section{Transient activation of the culture induces long-lasting changes in network activity}

Hippocampal neurons obtained from postnatal day 0 rat hippocampus and grown in drug-free dissociated culture conditions express spontaneous network activity, consisting of mixed excitatory and inhibitory synaptic currents that develop over 7-8 d into coordinated rhythmic bursts of activity, as seen elsewhere (Verderio et al., 1999). Cells grown in a medium containing the NMDA antagonist APV showed higher frequency of large bursts and small interburst synaptic events $(0.72 \pm 0.08$ and $10.05 \pm 0.97$ $\mathrm{Hz}$, respectively) by comparison to cells in control cultures $(0.26 \pm 0.05$ and $4.8 \pm 0.75 \mathrm{~Hz})$. This difference was attributable to the growth of the culture in APV and not to the presence of the drug in the recording medium, because exposure of control cultures to APV did not change their activity patterns (data not shown). Exposure to a CM (APV- and $\mathrm{Mg}$-free) for $3 \mathrm{~min}$ induced a lasting change in activity in the APV-grown cultures; synaptic events in the burst became larger (Fig. 1) and more organized: enhancement of network activity persisted for as long as recording was possible (up to $1 \mathrm{hr}$, data not shown). Such long-term changes were not seen in control cultures (Fig. 2). The change in network activity could be expressed as an increase of mean synaptic current, increase in amplitude, and/or frequency of bursts and an increase of cross-correlation between cells. A sig- 
a

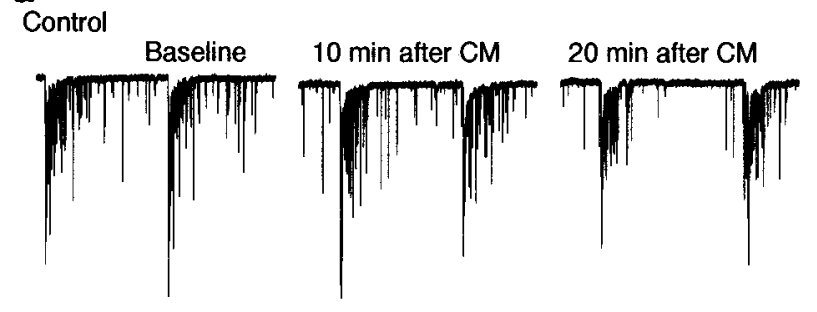

APV-grown

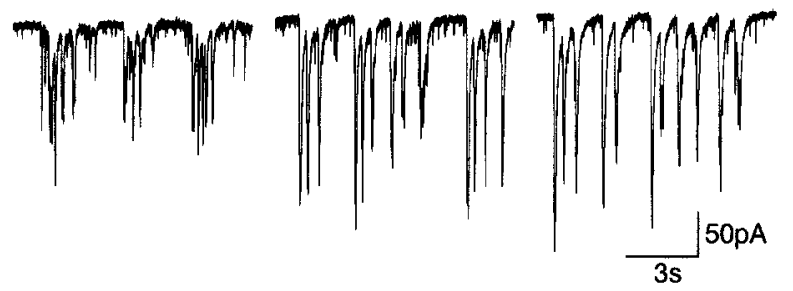

b

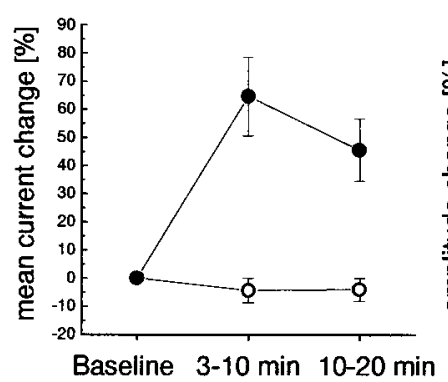

C

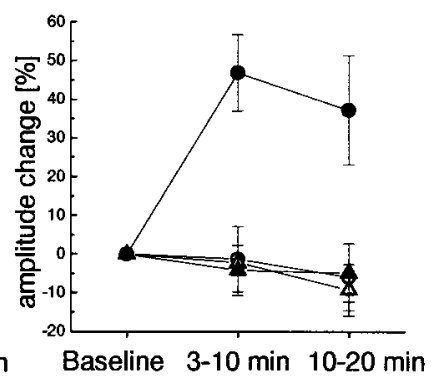

Figure 2. Persistent modification of network activity is dependent on growth conditions of the cultures. $a$, Sample records taken (from left to right) before, within $10 \mathrm{~min}$, and $20 \mathrm{~min}$ after exposure to the CM. Top, Cell grown in control conditions; bottom, cell grown in APV-containing growth medium. $b, c$, Summary of cells analyzed between 3 and $10 \mathrm{~min}$ and 10 and $20 \mathrm{~min}$ after the CM (control, open symbols, $n=5$; APVgrown, filled symbols, $n=9$ ). The mean synaptic current and burst amplitude (circle) increased after treatment only in APV-treated culture. Despite the increase in burst amplitude, the amplitude of interburst events did not change (triangles).

nificant $(50.8 \pm 10.7 \%)$ increase of mean synaptic current in APV-grown cultures was observed between 3 and 20 min after wash in 14 of 17 cells tested. In contrast, only one of nine cells showed an increase in mean current in control culture, and three of nine showed a decrease (on average, $-9.9 \pm 7.6 \%$ ).

To determine the temporal course of changes, mean synaptic currents per $15 \mathrm{sec}$ intervals and amplitude of bursts were measured between 3 and 10 min and between 10 and 20 min after replacement of the $\mathrm{CM}$ by the standard recording medium ( $n=$ 9 and 5 in APV-grown and control culture, respectively; Fig. 2). The mean current in APV-treated culture increased by $64.9 \pm$ $14.1 \%$ and by $45.8 \pm 11.2 \%$ between 3 and 10 min and 10 and 20 min, respectively, whereas in control culture, only one cell showed a change, and the average was $-4.3 \pm 4.3 \%$ and $-4 \pm 4 \%$, respectively (Fig. 2). Measuring the burst peak amplitude in the same cells showed an increase of $46.8 \pm 9.9 \%$ and $37.2 \pm 14.1 \%$ in APV-grown culture, whereas on average control culture showed a change of $-1.3 \pm 8.4 \%$ and $-5.9 \pm 8.7$ (Fig. $2 c$ ). In contrast, the amplitude of the interburst events (small individual synaptic events probably representing single EPSCs) did not change at any time after exposure to the CM (Fig. $2 c$ ), indicating a
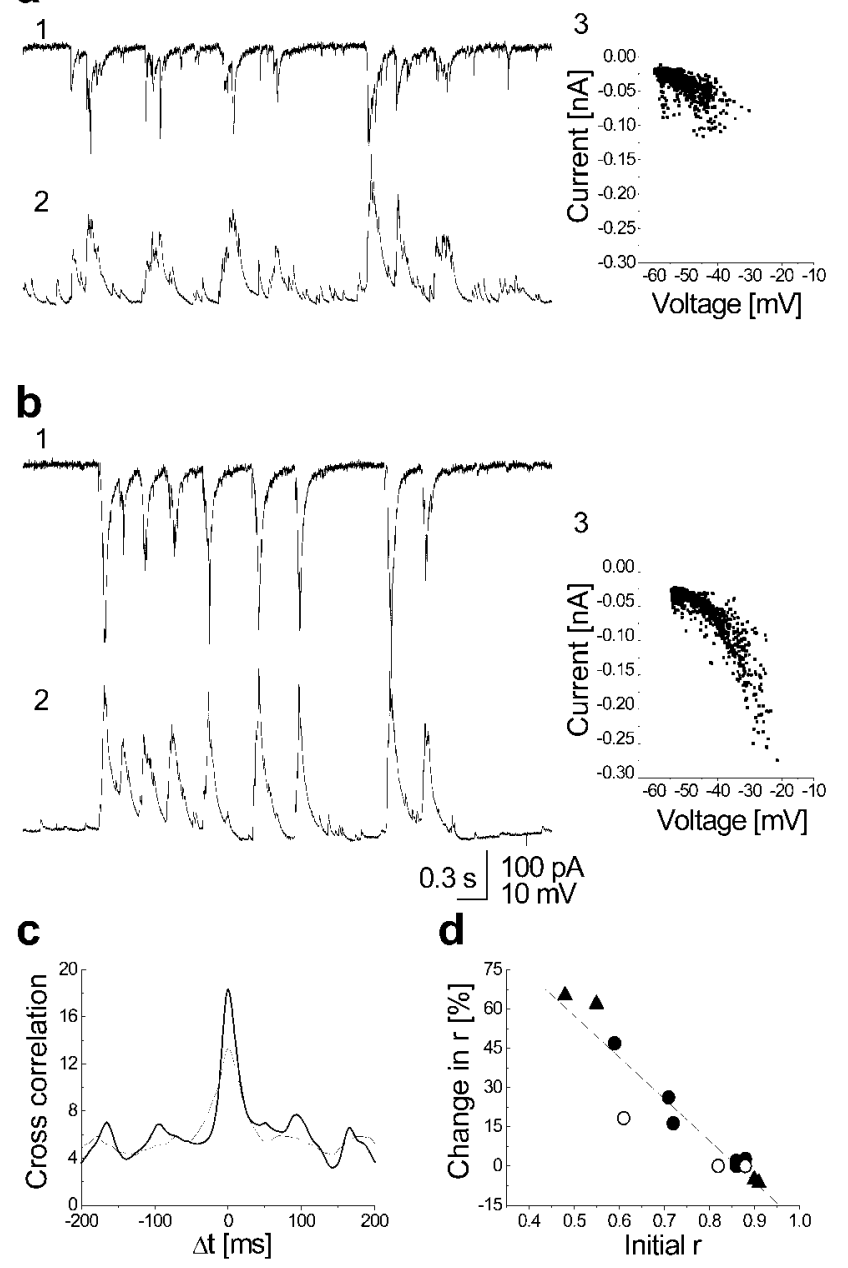

Figure 3. CM increases the correlation between cells proportionally to their initial correlation value. $a, b$, Voltage-clamp (top trace, 1 ) and current-clamp (bottom trace, 2) recording from two adjacent cells and their current versus voltage plot with the linear fit (3), before $(a)$ and after $(b)$ treatment. After the treatment, besides increasing its amplitude, the signal became less "noisy", and there is an increase in correlation coefficient of the linear fit between the two cells from 0.77 to 0.88 (the median of the experiment is from $r=0.72-0.84$ ). $c$, Cross-correlation between the two traces, normalized to their area so that the increase in the amplitude does not affect it, became narrower and bigger after the treatment (thicker line). $d$, Plot of percentage change of correlation coefficient $r$ versus initial value of $r$ of all double recordings: control (open circle), APV-treated cells (closed circle), and APV-treated cells plus Mg (triangle). $r$ before and after the treatment were calculated as a median of different trace sections (from 3 to 13 different sections; $9.8 \mathrm{sec}$ duration each), and the significance of the difference was tested (Wilcoxon test; $p<0.001$ ). The linear regression is of $r=0.94$.

that the network change may not be reflected in the properties of individual synapses.

One of the striking consequences of exposure to the CM was that activity in APV-grown culture was not only larger, but also became more synchronized. To investigate the degree of synchronicity, we performed double patch recording from adjacent cells in the culture, with one cell in voltage-clamp mode and a second in current-clamp mode. Usually the activity consists of both synchronous and asynchronous events (Fig. 3). After the treatment, besides an increase in their amplitude, all the events became synchronized (Fig. 3b,c). In general cells that were already 
a

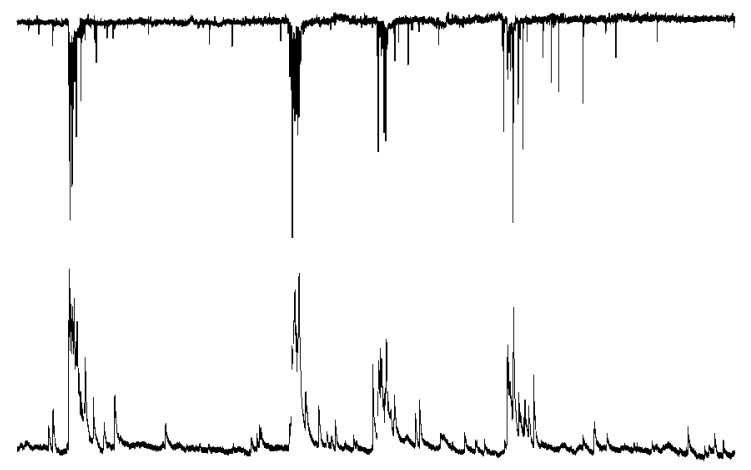

b
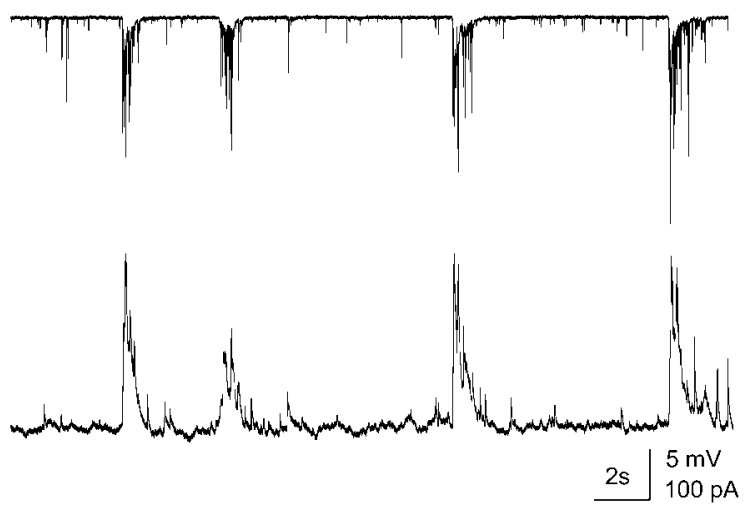

Figure 4. Buffering of intracellular calcium prevents an increase in the activity and in the correlation between cells. $a, b$, Double recording from adjacent cells in a culture incubated for $70 \mathrm{~min}$ with BAPTA-AM $(20 \mu \mathrm{M})$ before $(a)$ and after $(b) \mathrm{CM}$ exposure for $7 \mathrm{~min}$. Cells (voltage-clamp, top trace; current-clamp, bottom trace) still show network activity with both synchronous and asynchronous events. No change in activity or in the correlation coefficient was detected after perfusion with the CM.

organized and synchronous before the exposure to the CM did not show any change, independently from the condition in which they grown (Fig. 3d).

We then explored some parameters that may affect the change in network activity. The duration of the treatment beyond $3 \mathrm{~min}$ did not affect the magnitude of change, but when the protocol was applied for $<3 \mathrm{~min}$, no consistent effect was detected $(n=3)$. To investigate the role of activation of the NMDA receptor, a $\mathrm{Mg}$ free, CM-containing $100 \mu \mathrm{M}$ APV was applied. In none of three cases tested with this $\mathrm{CM}$ was there a persistent change in activity. In another set of experiments, APV-free, Mg-containing CM was applied. In three of six cells a small effect was detected. In one of these three cells the entire protocol was later applied with the $\mathrm{Mg}-\mathrm{APV}$-free $\mathrm{CM}$, and a further enhancement was observed. Thus, activation of the NMDA receptor is necessary for inducing the network change.

We then tested the involvement of changes in $\left[\mathrm{Ca}^{2+}\right] \mathrm{i}$ in the production of the long-term change in network activity. In three cultures pre-exposed to BAPTA-AM for $70 \mathrm{~min}(20 \mu \mathrm{M}$; which did not affect spontaneous network activity because this was similar to nonincubated culture), subsequent exposure to the CM failed to produce the long-term modification of the network activity (Fig. 4).

\section{Morphological analysis: postsynaptic changes}

Randomly selected, individual calcein-labeled neurons grown for at least $11 \mathrm{~d}$ in culture show little spontaneous motility or formation of novel spines, by comparison to younger cells (Papa et al., 1995; Ziv and Smith, 1996). The density of dendritic spines at this age was $45 \pm 5.3$ spines per $100 \mu \mathrm{m}$ dendrites in the control conditions, and $29.2 \pm 2.5$ spines per $100 \mu \mathrm{m}$ dendrites in the APV-grown cultures. These values are similar to densities of dendritic spines of cultured hippocampal neurons reported elsewhere (Papa et al., 1995; Murphy and Segal, 1996). A reduction in spine density in the presence of APV has also been seen before in young neurons (Collin et al., 1997). After exposure to the CM, which, in sister cultures produced long-term changes in network activity, cells began to express new protrusions, and modify existing ones, in a time-dependent manner. In an early series of experiments (our unpublished observations), we found that the presence of a robust electrophysiological change after exposure to the $\mathrm{CM}$ is a good predictor to a subsequent change in the morphology of sister cultures, and whenever such changes were not found, there were no changes in dendritic spines. Thus, although we did not systematically quantify the parallel changes in electrophysiology and morphology, further morphological experiments were conducted after verification that the culture can express long-term electrophysiological changes in network activity.

The new protrusions were divided into new filopodia, headless, mushroom, and stubby spines. Spines of all categories showed a significant increase in number over control values already at $1 \mathrm{hr}$ after exposure to the CM and continued to be formed within the following $2 \mathrm{hr}$ of observation (Fig. 5). Altogether, in 25 APVtreated cells (3892 $\mu \mathrm{m}$ length of dendrites) there were 27.0 new spines (with or without heads) per millimeter of dendrite, compared with 2.9 new spines per millimeter in a total of 13 control cells (2070 $\mu \mathrm{m}$ total length of dendrites). There were 20.0 additional new stubby spines per millimeter of dendrite in the APVtreated cells compared with 0.48 new stubby spines per millimeter of dendrite in the control cells. These differences were highly significant statistically. The number of new filopodia was small and not different between the treated and controls (Fig. $5 d$ ). The functionality of the novel spines was examined in a subset of neurons by labeling presynaptic terminals, at the end of the observation period (Fig. 5c). In total, 56 of 64 novel spines were adjacent to FM4-64-labeled terminals. This indicates that the novel spines are likely to be functional and receive input from viable terminals.

\section{Pruning of dendritic spines}

In addition to the formation of novel spines, we found quite a number of cases in which existing spines disappeared after exposure to the CM (Fig. 6). Altogether, 22.5\% of the total counted spines disappeared after exposure to the $\mathrm{CM}$ in the experimental group, by comparison to only $8.8 \%$ of the spines in the control neurons. This was further analyzed below.

\section{Morphological analysis: postsynaptic changes correlated with presynaptic activity}

In an attempt to trace down the origin of the novel innervated spines and the fate of the existing ones, we labeled presynaptic terminals at the beginning and the end of the experiment (Fig. $7 a, b)$. The goal of these experiments was to determine whether presence of a presynaptic terminal either on the shaft or on the spine affects the ability of the spine to change its morphology as 


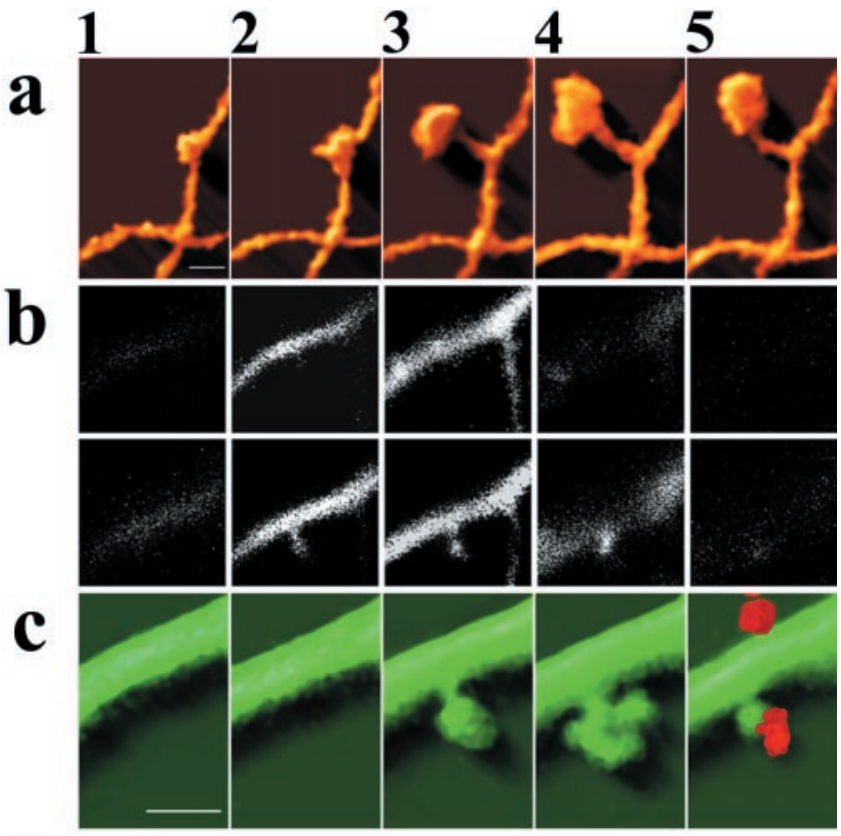

d

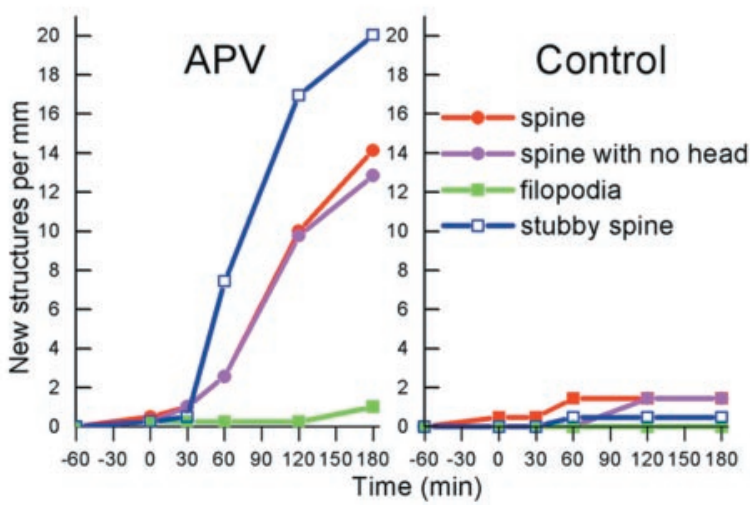

Figure 5. Exposure to a conditioning medium causes formation of novel dendritic spines in cultured hippocampal neurons. $a$ illustrates a dendritic spine, 3D-reconstructed at 30 min intervals, before $(1,2)$ and after $(3,4$, $5)$ exposure to the CM. $b$, Two series of $0.75-\mu$ m-thick optical sections of a dendritic segment used for the reconstruction of the images presented in $c$, illustrating the images shown in $c 2$, top, and $c 3$, bottom. A novel spine, not seen in $B$, top, is clearly seen in $B$, bottom. $c$ illustrates a time-lapse, 3D image of a dendrite from a neuron in a culture that was exposed at the end of the imaging sequence to a medium containing FM4-64, to label presynaptic terminals (c5). Note that after exposure to the FM4-64containing medium (high $\mathrm{K}$, depolarizing medium) the spine head shrinks. Cells were grown in APV, injected with calcein, and exposed to the $\mathrm{CM}$ in the recording chamber for $4 \mathrm{~min}$, followed by an extensive wash, as detailed above. Scale bars: $A, C, 1 \mu \mathrm{m}(2.5 \mu \mathrm{m}$ in $B) . D$, Summary diagram of the formation of novel dendritic spines in the experimental (left) and control groups (right) depicting different types of spines, including headed spines, spines with no head, stubby spines, and filopodia. Spines of the first three categories are formed within $2 \mathrm{hr}$ of exposure to the CM, whereas no similar change was seen in the control cultures, exposed to the same CM. The difference between control and experimental groups of neurons is highly significant $(t$ test for comparison between control and APV-treated cells yielded $p<0.01$.

a result of exposure to the CM. As opposed to the previous study, in which only new spines were counted, in the present study all spines were counted and categorized into spines that were maintained or disappeared after exposure to the CM. The results are summarized in Figure $7 c$, comprising a total of 16 cells $(3543 \mathrm{~nm}$ total dendritic length) exposed to the CM. Strikingly, spines that were not innervated before had a significantly higher tendency to disappear after the exposure to the $\mathrm{CM}$ by comparison to innervated spines, which tended to maintain their shape. Thus, exposure to a CM promotes the pruning of noninnervated spines. This is by no means an all-or- none effect, because there are many spines not associated with an FM4-64 terminal that were maintained across the measurement time. Finally, a detailed analysis of the novel spines indicated that in most cases studied, novel spines were formed where previously there was an FM4-64 bouton adjacent to a dendritic shaft, $(n=232)$ compared with cases in which a novel spine was formed where there was no detectable FM4-64 bouton beforehand $(n=119)$. Thus, the presence of a putative shaft synapse facilitates formation of a novel spine.

\section{DISCUSSION}

The present results demonstrate that an acute exposure of cultured hippocampal neurons, grown in presence of an NMDA receptor antagonist, to a conditioning medium, which favors activation of the NMDA receptor, can produce a lasting change in their network activity. This change is expressed as an increase in the size of AMPA receptor-mediated EPSCs as well as in the synchronization among cells in the culture. The network changes are followed by morphological plasticity, including formation of novel dendritic spines that are in close proximity to viable presynaptic terminals and pruning of existing spines. Thus, a correlation between functional and morphological plasticity can be demonstrated in dissociated hippocampal neurons in culture, which encourages further experiments on the mechanisms of morphological plasticity.

The cellular basis of the long-term change in network activity is not clear as yet. It involves activation of the NMDA receptor, as it was not found when the culture was exposed to $\mathrm{Mg}$-free, APV-containing medium. It involved a rise of intracellular calcium concentration and was blocked by BAPTA, as seen with classical LTP, but whether the change is at the synapse, involving presynaptic (Ma et al., 1999) or postsynaptic (Liao et al., 1995) changes or changes in excitability of the neurons is not entirely clear. In preliminary experiments we could not find lasting changes in miniature synaptic currents recorded in cells that expressed the change in network activity, but this result may just indicate that only a subset of synapses are changed, and the change is diluted among many active synapses. Likewise, we did not find a change in firing threshold (our unpublished observations), but the possibility of a change in excitability of the cells cannot be ruled out. Finally, although we do observe changes in the AMPA-mediated excitatory synaptic currents, recorded in presence of APV, we do not exclude the possibility that inhibitory synaptic currents are also enhanced by the CM. We did not specifically address this issue in the present study, but it is likely that an enhanced network activity will also involve a rise in activation of the inhibitory neurons in the network. A change in network may thus reflect small changes in many participating neurons, which may not be detected otherwise, but will result in an amplified reverberating activity of the entire network. This reverberating, ongoing activity will enhance detection of morphological changes in neurons of the network.

A major issue in the understanding of the role of dendritic spine in neuronal plasticity involves the rules governing formation and pruning of dendritic spines and the relevance of these processes to the functioning of the synapse. Although there is evidence to indicate that spines are formed from filopodia, after formation of a synapse with axon terminal in vitro (Jontes and 

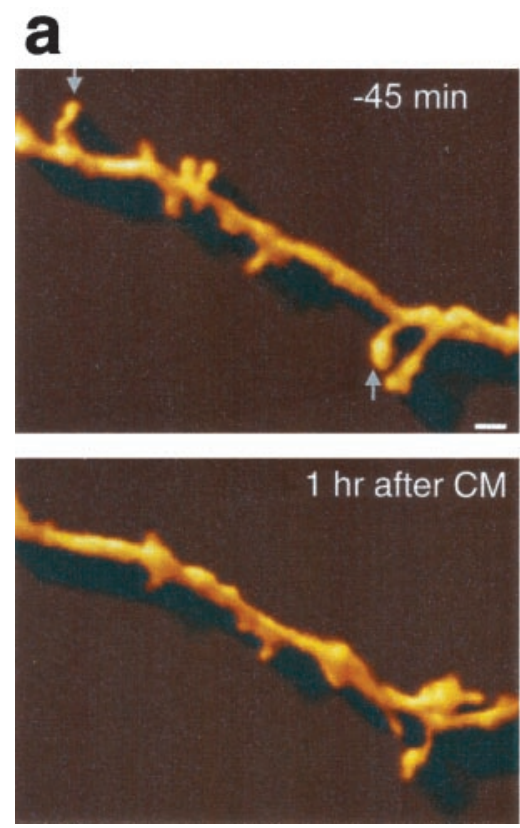
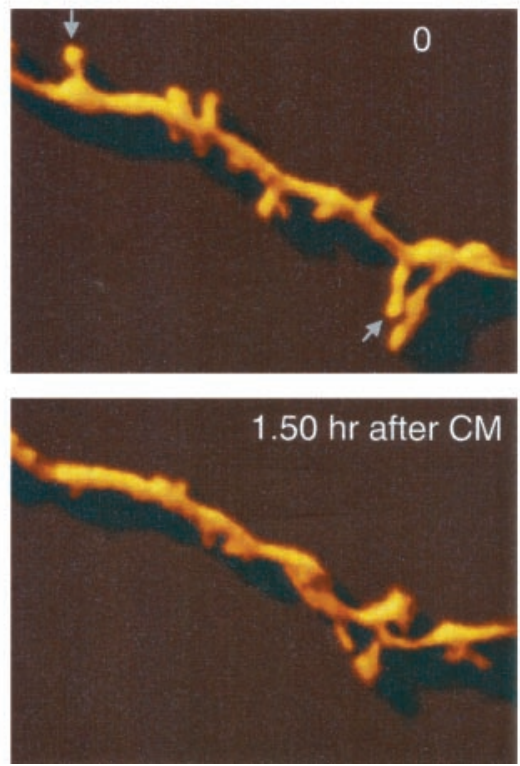

b

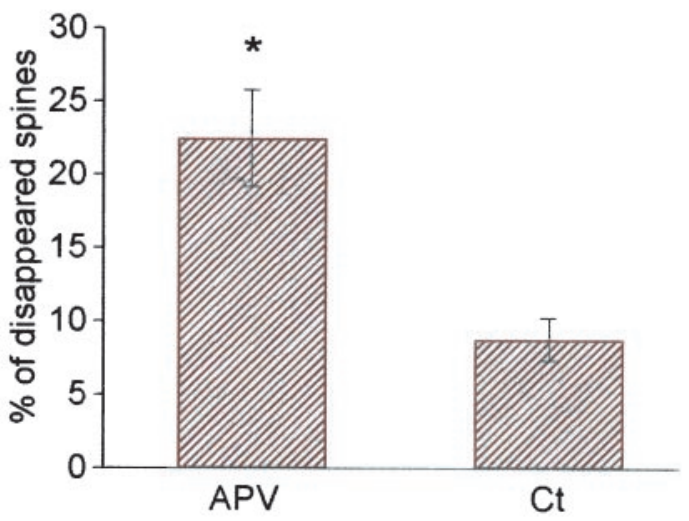

Figure 6. Selective spine pruning after exposure to the CM. $a$, A sequence of 3D images of a dendrite taken at various times before and after exposure to the CM (at time 0). Many, but not all the spines on this dendrite disappear in the course of the experiment, after exposure to the CM. $b$, A summary comparison between control $(\mathrm{Ct})$ and APV-treated cultures, illustrating that the latter group has a significantly higher proportion of disappearing spines of the total population sampled, than control groups. After exposure to the CM, $22.5 \%$ of the spines disappear in the APV-treated culture, compared with only $8.8 \%$ of the spines in the control cultures $(t$ test, $p<0.003)$. Scale bar, $1 \mu \mathrm{m}$.

Smith, 2000), this may not be the only or even the main mechanism for formation of a spine. Fiala et al. (1998) suggested that filopodia may be involved in drawing presynaptic terminals to dendritic shaft, to cause formation of shaft synapses, which will eventually form spines, as suggested here. Clearly, in the mature neuron, where filopodia are not abundant, spine density can vary by $35 \%$ across the estrus cycle (Woolley and McEwen, 1993), indicating that novel spines may evolve from a shaft synapse. In few cases in the present study, we observed spine head branching (Figs. $6 a, 7 b$ ), which may constitute another mode of formation of novel spines (Sorra et al., 1998; Toni et al., 1999), but most of them seem to grow off the dendritic shaft. This growth may be facilitated by the presence of an active synapse on the shaft, but the signal for the formation of a spine from a shaft synapse over several minutes is not known. Likewise, it is not entirely clear what might be the functional significance of the formation of a spine from a shaft synapse. Unlike previous studies (Engert and Bonhoeffer, 1999), we were able to show that novel spines are associated with FM4-64-labeled terminals and that there is a clear relation between the fate of a spine and its association with a viable terminal. Still, the fact that synaptic activity, recorded in the soma, increases after a conditioning protocol does not necessarily mean that the novel spine actually contributed to this enhanced activity, which can be performed by existing spineshaft synapses just the same. The difference between the impact produced by shaft and spine synapses on the electrical response recorded in the soma is still unknown. A role of spine neck length in regulation of calcium responses in the spine head relative to the parent dendritic shaft has been proposed recently (Volfovsky et al., 1999), and changes in spine length may contribute to the unique features of a spine relative to a shaft synapse.

The enhanced network activity is associated with both formation of novel spines and pruning of existing ones. Whereas formation of novel spines has long been associated with enhanced synaptic activity (Harris and Kater, 1994), pruning of existing spines has only recently been associated with enhanced excitation both in vivo and in vitro, as indicated by its blockade by APV (Bock and Braun, 1999). In fact, a very large enhancement of activity in a hippocampal network, associated with an epileptic seizure, can lead to a net pruning of dendritic spines (Jiang et al., 1998), as suggested recently (Segal et al. 2000). Thus, the simultaneous formation of novel spines, and pruning of existing ones, as is the case with a moderate increase in activity, may lead to little or no apparent change in the total number of spines. Instead, an increase in the number of spines associated with an active presynaptic terminal can be seen. As suggested elsewhere (Ma et al., 1999), it is possible that the FM4-64 only stains a subset of active synapses, and that this subset can grow in size after a conditioning protocol. In preliminary experiments, we immunostained the cultures with anti-synaptophysin antibodies, and we found that indeed most if not all of the FM4-64 particles are stained for synaptophysin, but that a sizable fraction of synaptophysin particles are not stained with FM4-64. The reason for this discrepancy is not known, but it may explain the persistence of spines in the absence of viable FM4-64-stained terminals. Further experiments are needed to analyze this possibility.

The pruning seen in the present experiments, in spines not associated with an active presynaptic terminal, can still result from a rise of intracellular calcium, caused by backpropagating action potentials and synaptic current spread from adjacent synapses. We (Volfovsky et al., 1999) have shown that a calcium wave coming from the soma can invade the spine, especially if it is a short one. Thus, spine pruning may reflect a Hebbian rule, predicting that a spine may disappear if postsynaptic activity in it is not correlated with presynaptic activity.

The time course of formation and pruning of dendritic spines seen in the present study corresponds to a similar time course of spine formation seen in a cultured slice (Engert and Bonhoeffer, 

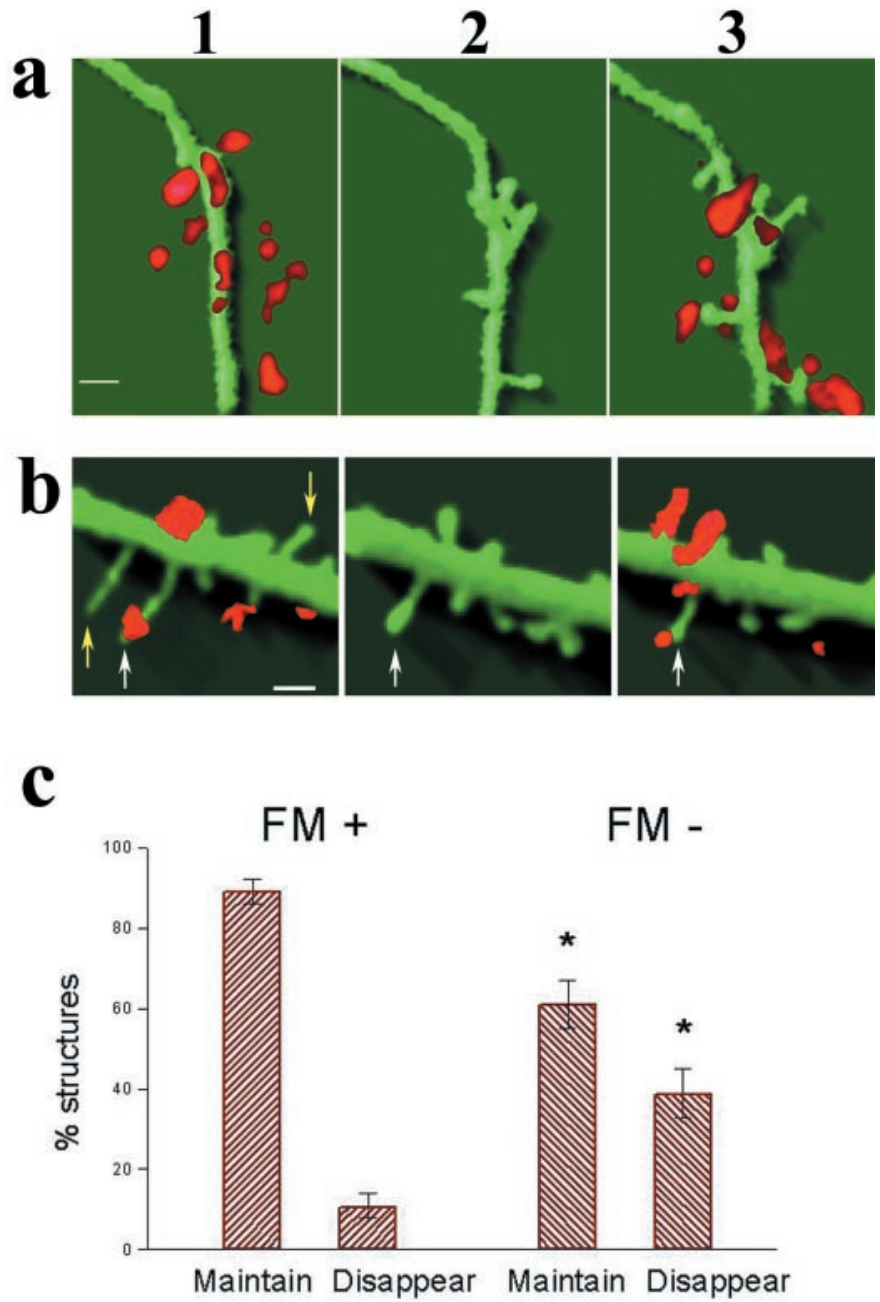

Figure 7. Dynamic changes in dendritic spines are predicted by the presence of active presynaptic terminals. $a, b$, Two illustrations of dendrites and spines stained with FM4-64 before and after exposure to the CM. In $a$, several FM-labeled puncta are seen near the 3D-reconstructed dendrite, which, before the start of the experiment, did not contain any clear spines. Within 90 min after exposure to the CM, several spines are formed and are eventually associated 30 min later, with FM4-64-stained particles. In $b$, existing spines are seen already at the start of the experiment, some of which are associated with an FM4-64-labeled particles. After to the CM two spines, not associated with FM, disappear (yellow arrows) and the rest (white arrows) are maintained. $c$, The maintenance of dendritic spines is correlated with the presence of FM particles. The population of dendritic spines was divided into spines linked and not linked to FM. Most of those linked to FM particles (FM+) were maintained (16.3 spines/100 $\mu \mathrm{m}$ dendrites maintained compared with 1.77 spines disappearing), whereas a large proportion of spines not associated with FM (FM-) disappeared during the experiment (8.36 spines/100 $\mu \mathrm{m}$ were maintained, compared with 5.41 spines/100 $\mu \mathrm{m}$ dendrites disappearing). The difference between the labeled and nonlabeled spines was highly significant ( $t$ test; $p<0.001$ ). Scale bars: $a, 2 \mu \mathrm{m} ; b, 1 \mu \mathrm{m}$.

1999), and to the time course of formation of novel synapses (Vardinon-Friedman et al., 2000). Thus, whereas the morphological change cannot account for the initial electrophysiological elevation of network activity, it can certainly underlie the late maintenance of the enhanced activity. In a way, the CMenhanced network activity can be visualized as a sped-up maturation process because older cultures maintain a more organized network activity and a higher density of mature dendritic spines (Papa et al., 1995), supporting the general proposal that a matu- ration process uses similar rules as those found after an LTP protocol (Ben-Ari et al., 1997).

The induction of long-term changes in patterned synaptic activity seen here was selective to the APV-treated cultures, although both cultures were exposed to the same medium, which enhances activation of the NMDA receptor. One possible explanation for this selective effect is that the growth of the cells in the presence of blocked NMDA receptors may result in upregulation of the NMDA receptors, to allow much more calcium influx into the affected cells compared with controls. This possibility has been alluded to before (Segal and Furshpan, 1990). As seen elsewhere (Collin et al., 1997), growth of cultured hippocampal slices in APV reduces spine density, but when APV is washed away, LTP is enhanced in these slices compared with controls. The opposite possibility, that dendritic spines actually are formed in the presence of APV, has been suggested before (Rocha and Sur, 1995), but this may involve a unique type of sparsely spiny thalamic neurons studied there. At any rate, although the NMDA receptor does not play a crucial role in formation of synaptic networks, it does affect maturation of these networks, as predicted by others (Liao et al., 1995; Ben-Ari et al., 1997)

The relation between production of LTP and an increase in network activity is not entirely clear. Whereas most of the studies on LTP are focusing on the first EPSP produced by single pulse stimulation, there is little evidence that an increase in single EPSP is relevant to the ongoing activity in a network (Markram and Tsodyks, 1996). In fact, enhanced synaptic activity may cause scaling down of the network activity (Turrigiano and Nelson, 2000). Thus, a tetanic stimulation evoking LTP may not be the most efficient stimulation to evoke a network change. Our results indicate that network activity can be changed, and the rules governing these changes, i.e., dependence on activation of the NMDA receptor, and on influx of calcium, are similar to those studied in the isolated EPSP case. Thus, the long-term enhancement of the behavior of the network, and not necessarily of the individual synapse, is associated with both formation of novel functional spines and pruning of existing ones.

\section{REFERENCES}

Ben-Ari Y, Khazipov R, Leinekugel X, Caillard O, Gaiarsa JL (1997) GABAA, NMDA and AMPA receptors: a developmentally regulated "menage a trois". Trends Neurosci 20:523-529.

Bock J, Braun K (1999) Blockade of $N$-methyl-D-aspartate receptor activation suppresses learning-induced synaptic elimination. Proc Natl Acad Sci USA 96:2485-2490.

Collin C, Miyaguchi K, Segal M (1997) Dendritic Spines in cultured hippocampal slices: correlating structure and function. J Neurophysiol 77:1614-1623.

Dunaevsky A Tashiro A Majewska A Mason C R Yuste (1999) Developmental regulation of spine motility in the mammalian central nervous system. Proc Natl Acad Sci USA 96:13438-13443.

Engert F, Bonhoeffer T (1999) Dendritic spine changes associated with hippocampal long-term synaptic plasticity. Nature 399:66-70.

Fiala JC, Feinberg M, Popov V, Harris KM (1998) Synaptogenesis via dendritic filopodia in developing hippocampal area CA1. J Neurosci 18:8900-8911.

Fischer M, Kaech S, Knutti D, Matus A (1998) Rapid actin based plasticity in dendritic spines. Neuron 20:847-854.

Halpain S, Hipolito A, Saffer L (1998) Regulation of F-actin stability in dendritic spines by glutamate receptors and calcineurin. J Neurosci 18:9835-9844.

Harris KM, Kater SB (1994) Dendritic spines: cellular specializations imparting both stability and flexibility to synaptic function. Annu Rev Neurosci 17:341-371.

Jiang M, Lee CL, Smith KL, Swann JW (1998) Spine loss and other persistent alterations of hippocampal pyramidal cell dendrites in a model of early-onset epilepsy. J Neurosci 18:8356-8368.

Jontes JD, Smith SJ (2000) Filopodia, spines and generation of synaptic diversity. Neuron 27:11-14

Korkotian E, Segal M (1999) Release of calcium from stores alters the 
morphology of dendritic spines cultured hippocampal neurons. Proc Natl Acad Sci USA 96:12068-12072.

Liao D Hessler NA, Malinow R (1995) Activation of postsynaptic silent synapses during pairing-induced LTP in CA1 region of hippocampal slices. Nature 375:400-404.

Luscher C, Nicoll RA, Malenka RC, Muller D (2000) Synaptic plasticity and dynamic modulation of the postsynaptic membrane. Nat Neurosci 3:545-550.

Ma L, Zablow L, Kandel ER, Siegelbaum SA (1999) Cyclic AMP induces functional presynaptic boutons in hippocampal CA3-CA1 neuronal cultures. Nat Neurosci 2:24-30.

Malgaroli A, Tsien RW (1992) Glutamate-induced long-term potentiation of the frequency of miniature synaptic currents in cultured hippocampal neurons. Nature 357:134-139.

Markram H, Tsodyks M (1996) Redistribution of synaptic efficacy between neocortical pyramidal neurons. Nature 382:807-810.

McKinney AR, Capogna M, Durr R, Gahwiler BH, Thompson SM (1999) Miniature synaptic events maintain dendritic spines via AMPA receptor activation. Nat Neurosci 2:44-49.

Murphy DD, Segal M (1996) Regulation of dendritic spine density in cultured rat hippocampal neurons by steroid hormones. J Neurosci 16:4059-4068.

Papa M, Bundman MC, Greenberger V, Segal M (1995) Morphological analysis of the development of dendritic spines in primary cultures of hippocampal neurons. J Neurosci 15:1-11.

Rocha M, Sur M (1995) Rapid acquisition of dendritic spines by visual thalamic neurons after blockade of $N$-methyl-D-aspartate receptors. Proc Natl Acad Sci USA 92:8026-8030.

Segal MM, Furshpan EJ (1990) Epileptiform activity in microcultures containing small numbers of hippocampal neurons. J Neurophysiol 64:1390-1399.

Segal M, Korkotian E, Murphy DD (2000) Dendritic spine induction and pruning-common cellular mechanisms? Trends Neurosci 23:53-57.

Sorra KE, Fiala JC, Harris KM (1998) Critical assessment of the involvement of perforations, spinules, and spine branching in hippocampal synapse formation. J Comp Neuron 398:225-240.

Tao HZ, Zhang LI, Bi GQ, Poo MM (2000) Selective presynaptic propagation of Long-Term Potentiation in defined neural networks. J Neurosci 20:3233-3243.

Toni N, Buchs PA, Nikonenko I, Bron CR, Muller D (1999) LTP promotes formation of multiple spine synapses between a single axon terminal and a dendrite. Nature 402:421-425.

Turrigiano GG, Nelson SB (2000) Hebb and homeostasis in neuronal plasticity. Curr Opin Neurobiol 10:358-364.

Vardinon-Friedman H, Bresler T, Garner CC, Ziv N (2000) Assembly of new individual excitatory synapses: time course and temporal order of synaptic molecule recruitment. Neuron 27:57-69.

Verderio C, Bacci A, Coco S, Pravettone E, Fumagalli G, Matteoli M (1999) Astrocytes are required for the oscillatory activity in cultured hippocampal neurons. Eur J Neurosci 11:2793-2800.

Volfovsky N, Parnas H, Segal M, Korkotian E (1999) Geometry of dendritic spines affects calcium dynamics in hippocampal neurons: theory and experiments. J Neurophysiol 82:450-462.

Woolley CS, McEwen BS (1993) Roles of estradiol and progesterone in regulation of hippocampal dendritic spine density during the estrous cycle in the rat. J Comp Neurol 336:293-306.

Ziv NE, Smith SJ (1996) Evidence for a role of dendritic filopodia in synaptogenesis and spine formation. Neuron 17:91-102. 